TITLE:

\title{
Morphologic and functional changes in retinal vessels associated with branch retinal vein occlusion.
}

\section{AUTHOR(S):}

Muraoka, Yuki; Tsujikawa, Akitaka; Murakami, Tomoaki; Ogino, Ken; Kumagai, Kyoko; Miyamoto, Kazuaki; Uji, Akihito; Yoshimura, Nagahisa

\section{CITATION:}

Muraoka, Yuki ... [et al]. Morphologic and functional changes in retinal vessels associated with branch retinal vein occlusion.. Ophthalmology 2013, 120(1): 91-99

\section{ISSUE DATE:}

2013-01

URL:

http://hdl.handle.net/2433/169954

\section{RIGHT:}

(c) 2013 American Academy of Ophthalmology. Published by Elsevier Inc.; この論文は出版社版でありません。引用の際には出版社版をご確 認ご利用ください。; This is not the published version. Please cite only the published version. 
1 Morphological and Functional Changes in Retinal Vessels Associated with Branch

2 Retinal Vein Occlusion

3 Running head: Changes in Retinal Vessels due to BRVO

4

5 Yuki Muraoka, MD, Akitaka Tsujikawa, MD, Tomoaki Murakami, MD, Ken Ogino, MD,

6 Kyoko Kumagai, MD, Kazuaki Miyamoto, MD, Akihito Uji, MD, and Nagahisa Yoshimura,

$7 \quad M D$

8 Department of Ophthalmology and Visual Sciences, Kyoto University Graduate School of

9 Medicine, Kyoto, Japan.

11 This article contains online-only material.

12 The following should appear online only: Figures 2, 3, 5, 8.

14 This study was supported in part by the Japan Society for the Promotion of Science

15 (JSPS), Tokyo, Japan (Grant-in-Aid for Scientific Research, no. 21592256), and the

16 Japan National Society for the Prevention of Blindness, Tokyo, Japan.

18 Conflict of interest: No authors have any financial or conflicts of interests to disclose. 
1 Address correspondence to: Akitaka Tsujikawa, MD, Department of Ophthalmology,

2 Kyoto University Graduate School of Medicine, Sakyo-ku, Kyoto 606-8507, Japan.

$3 \quad$ E-mail: tujikawa@kuhp.kyoto-u.ac.jp 
1

\section{Abstract}

Objective: To study the morphological and functional changes in retinal veins of eyes affected with branch retinal vein occlusion (BRVO) by thin sectioning with optical coherence tomography (OCT).

Design: Prospective, observational, cross-sectional study.

Participants: Twenty-five consecutive patients (25 eyes) with acute BRVO.

Methods: Major retinal veins, arteries, and arteriovenous (A/V) crossing were examined by sequential thin sectioning by Spectralis HRA+OCT. The retinal blood flow was mimicked in vitro and was scanned with Spectralis HRA+OCT.

Main Outcome Measures: Morphological characteristics of normal and BRVO-affected retinal vessels seen in OCT sections.

Results: Cross-sectional OCT images revealed physiological retinal vessels as oval-shaped configurations with 4 distinctive hyperreflectivities in a line. The vessel walls showed the innermost and outermost hyperreflectivity, and the blood flow showed internal paired hyperreflectivities with an hourglass-shape. No eye with disturbed blood flow due to BRVO showed this internal hyperreflectivity pattern. In vitro, OCT sections of the blood within the glass tube without flow showed homogeneous reflectivities. Increased blood flow velocity resulted in the development of heterogeneous internal 
1 reflectivity and hourglass-shaped hyperreflectivities. In all eyes with acute BRVO,

2 sequential sectioning with OCT visualized three-dimensional vascular architecture as

3 well as the intravascular conditions at the $A / V$ crossing. At the affected $A / V$ crossing,

4 arterial overcrossing was seen in 17 eyes and venous overcrossing was seen in 8 eyes.

5 In eyes with arterial overcrossing, the retinal vein seemed to run deep under the artery at

6 the $A / V$ crossing, and the venous lumen often appeared to be preserved even at the $A / V$

7 crossing. In all eyes with venous overcrossing, the retinal vein appeared to be

8 compressed and choked between the internal limiting membrane and the arterial wall at

9 the $\mathrm{A} / \mathrm{V}$ crossing. OCT sectioning showed intravenous thrombi in 21 eyes, and the

10 thrombi were detected downstream of the $A / V$ crossing in all the cases. The detection

11 of thrombus was significantly associated with ischemic pattern in BRVO $(P=0.036)$.

12 Conclusion: In eyes with BRVO, sequential thin sections with OCT visualized

13 three-dimensional retinal vasculature. Present OCT findings suggest that BRVO may

14 occur by 2 different mechanisms, depending on the relative anatomical positions of

15 crossing vessels. 
1 Branch retinal vein occlusion (BRVO) is the second most common retinal vascular

2 disorder after diabetic retinopathy. BRVO is defined as focal occlusion of the major

3 retinal vein, occurring most frequently at an arteriovenous (A/V) crossing. ${ }^{1,2}$ Acute

$4 \quad$ BRVO is characterized by flame-shaped retinal hemorrhage and venous dilation and

5 tortuosity in the affected retina, often with macular edema. However, the precise

6 pathogenesis of BRVO is unclear. Previous histological studies have suggested that the

7 major artery and vein share a common adventitial sheath at the $A / V$ crossing and that the

8 mechanical compression of rigid arterial walls causes narrowing of the venous lumen,

9 resulting in flow disturbance at the $\mathrm{A} / \mathrm{V}$ crossing. ${ }^{3-5}$ A subsequent study with fluorescein

10 angiography (FA) reported that most of the eyes affected with BRVO showed venous

11 lesions downstream of the affected A/V crossing. ${ }^{6}$ The hemodynamic changes

12 downstream of the $A / V$ crossing were suggested to be important in the occurrence of

13 BRVO, which predisposes the eye to endothelial damage and thrombus generation. ${ }^{6,7}$

14 Technological advances in imaging resolution and acquisition speed of optical

15 coherence tomography (OCT) have enabled observation of the retinal architecture in

16 greater detail and have contributed to our understanding of the pathomorphology of

17 various macular diseases. Recent extensive investigations with OCT have

18 demonstrated the morphological changes associated with BRVO, including foveal cystoid 
1 spaces, ${ }^{8,9}$ serous retinal detachment,,${ }^{10,}{ }^{11}$ hyperreflective foci, ${ }^{12}$ and subretinal

2 hemorrhage..$^{13,14}$ In addition, OCT investigations revealed the importance of the outer

3 aspect of the foveal photoreceptor layer in the visual functions of eyes with BRVO. ${ }^{9,15-17}$

4 Based on these findings, OCT may have the potential to elucidate the morphological

5 changes and functional alterations in retinal veins affected with BRVO. To date,

6 however, little information is available on the OCT features of retinal vasculature. ${ }^{18-20}$

7 This study aimed to elucidate the pathogenesis of BRVO and investigate the clinical

8 relevance of the morphological changes of retinal vasculature in BRVO by studying the

9 morphological changes of BRVO-affected retinal veins by sequential thin sectioning with

10 OCT. 


\section{PATIENTS AND METHODS}

\section{Patients}

3 This prospective study consisted of 25 consecutive patients (25 eyes) with unilateral

4 acute major BRVO who were examined at the Department of Ophthalmology of Kyoto

5 University Hospital between August 2011 and March 2012. The duration of symptoms in

6 all the eyes was less than 6 months. BRVO was diagnosed on the basis of fundus

7 examination and FA findings of 2 retina specialists (AT, TM). Eyes with BRVO in which

8 the occluded site of the retinal vein was located within the optic disc or on the disc margin

9 were not included in the current study. Eyes with central retinal vein occlusion (CRVO),

10 co-existing ocular disease (i.e., glaucoma, diabetic retinopathy, or senile cataract that

11 resulted in poor-quality OCT images) were also excluded. In addition to the study

12 subjects, 9 patients ( 9 eyes) with old BRVO, in which the retinal hemorrhage had been

13 completely absorbed, and 1 patient (1 eye) with hemi-CRVO were also examined to

14 confirm the correlations between perfusion status obtained with FA and OCT findings.

15 The Institutional Review Board and the Ethics Committee of Kyoto University approved

16 this study. The study protocol adhered to the tenets of the Declaration of Helsinki. A

17 written informed consent for the study participation was obtained from each patient. 
1 best-corrected VA in a Landolt chart and fundus biomicroscopy with a non-contact lens,

$245^{\circ}$ digital fundus photographs were obtained using a digital fundus camera (TRC-50LX,

3 Topcon, Tokyo, Japan; 3,216 $\times 2,136$ pixels) after pupil dilatation. To assess the retinal

4 perfusion status, each patient underwent FA with a confocal laser scanning system

5 (HRA-2, Heidelberg Engineering, Heidelberg, Germany). Eyes with BRVO were

6 classified as ischemic when the area of nonperfusion was greater than 5 disc diameters

7 in size.

8 We assessed major retinal vessels within the retina affected with BRVO in detail

9 using sequential thin sectioning with Spectralis HRA+OCT (Heidelberg Engineering).

10 Sequential longitudinal sections were made along the major retinal veins or arteries

11 (minimally 25 sections of $5^{\circ}$ ). Sequential cross-sections were made vertical to each

12 major retinal vessel. Each section was averaged with at least 20 scans obtained at the

13 same location. The affected $\mathrm{A} / \mathrm{V}$ crossing was also examined with sequential

14 longitudinal and cross-sections.

\section{OCT examinations of blood flow in vitro}

17 To mimic the retinal blood flow in vitro, we used fine glass tubes approximately $200 \mu \mathrm{m}$ in

18 diameter. Human blood was extracted from healthy volunteers and heparinized. A fine 
1 glass tube was connected to a pump to circulate the extracted human blood at arbitrary

2 flow rates. Cross-sections of the blood flowing within the tube were scanned using

3 Spectralis HRA+OCT. A 25-diopter lens was attached to an OCT machine to adjust the

4 focus on the blood flow within the glass tube. Each image was obtained using an

5 average of 20 scans. Previous studies have shown that the flow velocity in the major

6 retinal vessels of healthy eyes is approximately $5-35 \mathrm{~mm} / \mathrm{s}^{21}$ To cover the physiological

7 and pathological flow velocity, the flow rate (blood velocity) in the glass tube was

8 gradually increased from $0-10.0 \mathrm{ml} / \mathrm{h}(0-88.4 \mathrm{~mm} / \mathrm{s})$.

\section{Statistical Analysis}

11 Statistical analysis was performed using PASW Statistics version 18.0 (SPSS, Chicago,

12 IL). Values are presented as mean \pm standard deviation. For statistical analysis, VA

13 measured with a Landolt chart was converted to a logarithm of the minimum angle of

14 resolution (logMAR). Student's $t$ test was used to compare quantitative data with normal

15 distributions and equal variance. Significant differences in the sampling distributions

16 were determined using the chi-square test. A $P$ value of less than 0.05 was considered

17 to be statistically significant. 
1 RESULTS

2 OCT imaging of normal retinal vessels

3 To study the OCT features of retinal vasculature, we first examined the major retinal

4 vessels in the healthy contralateral eyes. Cross-sectional OCT images revealed major

5 retinal vessels as oval-shaped configurations with heterogeneous reflectivities, mainly in

6 the retinal nerve fiber layer and occasionally in the inner plexiform layer (IPL) (Fig. 1). In

7 cross-sections, physiological vessels showed 4 distinctive hyperreflectivities in a line.

8 The top and bottom of the vessel walls, which were vertical to the OCT light source,

9 showed the innermost (vitreous side) and outermost (retinal pigment epithelium (RPE)

10 side) hyperreflectivities. The arterial walls generally had higher reflectivity as compared

11 to the venous walls. All retinal vessels with physiological blood flow showed inside

12 paired hyperreflectivities, which was frequently hourglass-shaped (Fig. 1). The internal

13 paired hyperreflectivities showed a double "c" pattern when the blood flow converged

14 from 2 different veins (right vein shown in Fig. 1C).

Figure 2 (available at http://aaojournal. org) shows the longitudinal OCT sections of

16 the major retinal vessels. Consistent with the cross-sections of the major retinal vessels,

17 retinal vessels had 4 hyperreflective bands, of which the innermost and outermost bands

18 were derived from the vessel walls and the 2 intermediate bands were derived from the 
1 bloodstream.

3 Correlations between retinal blood flow in FA and OCT findings

4 To investigate whether the internal paired hyperreflectivities observed in the physiological

5 retinal vessels are derived from the retinal blood flow, 9 eyes with old BRVO were

6 examined with Spectralis HRA+OCT. The OCT images of the sheathed veins

7 associated with old BRVO were then compared with the blood flow seen in FA. While

8 blood flow was detected in the sheathed veins of 4 eyes, there was no blood flow in the

9 sheathed veins of 5 eyes. In OCT sections of sheathed retinal veins with no blood flow,

10 no internal paired hyperreflectivities were present (Fig. 3, available at http://aaojournal.

11 org). However, paired hyperreflectivities were detected in all 4 eyes with blood flow in

12 the sheathed veins.

14 OCT features of the blood flow in vitro

15 Figure 4 shows a fundus photograph and FA of a case of acute hemi-CRVO. In FA,

16 physiological blood flow is seen in the lower vein but the blood flow of the affected upper

17 vein is markedly disturbed. OCT cross-sections revealed internal paired

18 hyperreflectivities within the walls of the lower unaffected vein but homogeneous 
1 reflectivity without paired hyperreflectivities in the obstructed upper vein. samples through a fine glass tube at arbitrary flow velocities. Cross-sectional images of

4 human blood flowing within the tube were obtained with Spectralis HRA+OCT. Figure 5

5 (available at http://aaojournal. org) shows the OCT images of the blood flowing through

6 the fine glass tube at different flow velocities. OCT sections of the blood within the glass

7 tube without flow (at $0 \mathrm{~mm} / \mathrm{s}$ ) showed no paired hyperreflectivities. However, increased

8 blood flow velocity resulted in the development of heterogeneous internal reflectivity and

9 hourglass-shaped hyperreflectivities within the glass tube.

\section{Retinal vasculature changes associated with acute BRVO}

12 To investigate the changes in retinal vasculature associated with BRVO, 25 eyes of 25

13 patients (13 women and 12 men) with acute BRVO were examined with Spectralis

$14 \mathrm{HRA+OCT}$ (Table 1). Fundus examinations revealed retinal hemorrhage in all eyes, and

15 venous dilation and tortuosity in the affected retina. In all eyes, FA showed filling delay

16 in the affected vein but confirmed the blood flow at its late phase. OCT cross-sections

17 revealed paired hyperreflectivities in the unaffected retinal veins of all eyes. However,

18 none of the 25 eyes with BRVO showed this pattern of internal hyperreflectivities around 
1 the affected $A / V$ crossing. artery and vein at the affected $\mathrm{A} / \mathrm{V}$ crossing using fundus examination alone. In 3 of 25

4 eyes, even FA could not elucidate the relative anatomical position at the affected $\mathrm{A} / \mathrm{V}$

5 crossing. However, sequential thin sections of OCT clearly showed this association in

6 all 25 eyes. At the affected A/V crossing of our patients, arterial overcrossing was seen

$7 \quad$ in 17 eyes, while venous overcrossing was seen in 8.

9 examinations. Three-dimensional venous tortuosity and the condition of the venous

10 lumen at the affected $A / V$ crossing could be visualized by sequential longitudinal and

11 cross-sections analyzed by Spectralis HRA+OCT. In the eyes with BRVO of the artery

12 overcrossing, OCT findings revealed that the retinal vein ran deep under the artery at the

$13 \mathrm{~A} / \mathrm{V}$ crossing (Fig. 6). Of 17 eyes with arterial overcrossing, the venous wall remained in

14 the inner nuclear layer (INL) and the outer plexiform layer (OPL) in 6 eyes (35.3\%) and in

15 the outer nuclear layer (ONL) in 8 eyes (47.1\%). In 3 eyes (17.6\%), tortuous retinal

16 veins appeared to extend beyond the external limiting membrane (ELM) line and

17 approaching RPE. The distance between the deepest edge of the venous wall and RPE

18 ranged from $36-314$ (mean: $139.6 \pm 60.0) \mu \mathrm{m}$. In addition, OCT revealed that the 
1 venous lumen was generally preserved even at the A/V crossing. In contrast, in all eyes

2 with BRVO of the vein overcrossing, the affected vein appeared to be compressed and

3 choked between internal limiting membrane (ILM) and the arterial wall at the crossing

4 (Fig. 7).

$5 \quad$ In eyes with acute BRVO, OCT examination often showed thrombus formation

6 around the affected $\mathrm{A} / \mathrm{V}$ crossing. Thrombi were detected in 14 of 17 eyes with arterial

7 overcrossing (82.3\%) (Fig. 8, available at http://aaojournal. org), and in 7 of 8 eyes with

8 venous overcrossing (87.5\%) (Fig. 7). In 19 eyes, thrombus was seen from the A/V

9 crossing to the downstream (Fig. 7, Fig. 8, available at http://aaojournal. org). In 2 eyes,

10 the thrombi appeared to extend on both sides of the affected A/V crossing. None of the

11 eyes showed thrombi only at the crossing site or upstream from the site.

12 To determine the clinical relevance of thrombus formation, we evaluated its

13 association with clinical parameters (i.e., VA, foveal pathomorphology, and perfusion

14 status) (Table 2). The presence of thrombus was significantly correlated with the

15 perfusion status (ischemic BRVO) $(P=0.036$, chi-square test), and marginally correlated

16 with the height of foveal detachment $(P=0.083$, Student's $t$ test) and duration from the

17 onset of symptoms ( $P=0.070$, Student's $t$ test $)$. 


\section{DISCUSSION}

3 In cross-sectional OCT images of healthy eyes, major retinal vessels are observed as

4 oval-shaped configurations with heterogeneous reflectivities, often with 4 distinctive

5 hyperreflectivities in a line. The innermost and outermost hyperreflectivities are

6 interpreted to be derived from the vessel wall because the top and bottom of the vessel

7 walls are vertical to the OCT light source. All the vessels with physiological blood flow

8 show inside paired hyperreflectivities, which were frequently hourglass-shaped and

9 occasionally present in a double "c" pattern in convergent veins. However, no veins

10 without blood flow showed this feature. In addition, as shown in Figure 4 of a case of

11 acute hemi-CRVO, the vein with disturbed blood flow showed homogeneous reflectivity

12 without this feature. Therefore, we hypothesized that this intravascular feature is a

13 hallmark of the physiological blood flow.

14 To mimic the blood flow in the retinal vessels in vitro, we pumped blood samples

15 through a fine glass tube at various flow velocities (Fig. 5, available at http://aaojournal.

16 org). The cross-section of the glass tube without blood flow showed homogeneous

17 internal reflectivity. Increased blood flow velocity led to the development of

18 heterogeneous internal reflectivity and hourglass-shaped hyperreflectivities. According 
1 to previous reports with bidirectional laser Doppler velocimetry, the blood velocity is $7-35$

$2 \mathrm{~mm} / \mathrm{s}$ in the major retinal arteries and $5-25 \mathrm{~mm} / \mathrm{s}$ in the major retinal veins. ${ }^{21}$ With

3 unphysiological blood flow velocity $(88.4 \mathrm{~mm} / \mathrm{s})$, however, this feature disappeared and

4 the internal reflectivity was only observed at the anterior part of the lumen. When

5 applying the theory of rheology to the major retinal vessels, the central stream has the

6 maximal velocity; this was confirmed by Doppler OCT examinations, which revealed this

7 distribution of the intravascular flow velocity. ${ }^{22-24}$ However, it remains to be elucidated

8 why OCT sections of the retinal vessels with physiological blood flow show this feature.

9 It must be noted that this feature can be observed even if other OCT devices are used

10 (data not shown); this feature is not unique to Spectralis HRA+OCT alone.

Most cases of BRVO occur at A/V crossing sites. ${ }^{4,25-28}$ It has been suggested that

12 the large artery and vein share a common adventitial sheath at the A/V crossing.

13 Hypertension, arteriosclerosis, and old age are risk factors of BRVO. ${ }^{1,2,25}$ The

14 mechanical compression of rigid arterial walls has been suggested to cause narrowing of

15 the venous lumen, resulting in the concomitant flow disturbance at the $A / V$ crossing. In

16 a histopathological study conducted by Frangieh et al., ${ }^{3}$ all eyes with BRVO had fresh or

17 recanalized thrombi in the affected vein with varying degrees of arteriosclerosis of the

18 corresponding arteries. However, the cases investigated in their study were obtained 
1 after 8 months to 12 years after the onset of BRVO; furthermore, 6 of the 8 specimens

2 were obtained after the patients' death. In contrast, sequential thin sectioning of OCT

3 facilitates three-dimensional analysis of the retinal vasculature in the acute phase of

4 BRVO in vivo and elucidates the morphological changes as well as functional alterations

$5 \quad$ in the affected retinal veins.

$6 \quad$ Fundus examination revealed venous dilation and tortuosity in all eyes with acute

$7 \quad$ BRVO. OCT examinations revealed that venous tortuosity was more prominent in the

8 sagittal direction. In this study, the affected vein appeared to extend beyond the ELM

9 line, approaching the RPE lines in 3 eyes (17.6\%). Because Müller cells spread their

10 cell body from ILM to ELM and support the retinal architecture, ${ }^{29,30}$ the elongated retinal

11 veins in the eyes with BRVO may preferentially produce tortuosity in the sagittal direction

12 than in the frontal direction.

13 Previous clinical studies have reported that $85-100 \%$ of BRVO occurs at the arterial

14 overcrossing. . $^{3-7,28,31,32}$ Sequential thin sectioning with OCT allowed detailed

15 anatomical correlation between the artery and vein, revealing that BRVO occurred at the

16 arterial overcrossing in 17 of the eyes (68\%) investigated. Unexpectedly, OCT sections

17 of these eyes showed that the retinal vein runs deep below the artery and that the venous

18 lumen appears to be preserved at the A/V crossing. Our OCT findings are consistent 
1 with a previous histological report on normal eyes. ${ }^{5}$ Jefferies et al. reported that sharp

2 bending of the veins are frequently seen at the $A / V$ crossing without compression and

3 that focal narrowing of the veins are rarely seen at the A/V crossing in normal eyes. ${ }^{5}$

4 Recently, A/V sheathotomy has been reported as a treatment option for macular edema

5 associated with BRVO. ${ }^{26,33-35}$ Horio et al. ${ }^{35}$ reported that $A / V$ sheathotomy led to

6 transient improvement of the retinal blood flow but this improvement subsided at 1 month.

$7 \quad$ Although thrombi were occasionally observed to flow away through the affected vein

8 during the surgery, the effect of dissection of the common sheath would limit the

9 improvement in the circular disturbance. Mechanical compression of the artery may

10 play a less important role in the pathogenesis of BRVO at the arterial overcrossing site.

11 Rather, hemodynamic changes downstream of the A/V crossing may be essential in the

12 pathogenesis of BRVO.

13 Unexpectedly, current OCT examinations showed that BRVO occurred at the

14 venous overcrossing in 8 of the 25 eyes studied. In contrast to BRVO at the arterial

15 overcrossing, OCT revealed that the affected veins were compressed and choked

16 between ILM and the arterial wall at the venous overcrossing. At the venous

17 overcrossing, the mechanical compression caused by the ILM and the arterial walls at the

18 crossing would be primarily related to the onset of BRVO. To the best of our knowledge, 
1 however, limited information is available on the pathophysiology or clinical information of

2 eyes with this pattern of BRVO. Previous histological studies have reported that no

3 focal venous narrowing was present at the venous overcrossing in normal eyes and that

4 veins may deviate towards the vitreous. ${ }^{5}$ For the treatment of BRVO at the venous

5 overcrossing, it is difficult to perform A/V sheathotomy, but ILM peeling including the

6 affected $\mathrm{A} / \mathrm{V}$ crossing might effectively reduce the compression of the venous lumen.

8 affected with BRVO., ${ }^{3,4}$ However, in a previous report with FA, thrombus formation was

9 detected in $7 \%$ of eyes with BRVO, always downstream of the affected A/V crossing. ${ }^{6}$ In

10 the current study, thrombi were detected in 14 of 17 eyes with arterial overcrossing, and

11 in 7 of 8 eyes with venous overcrossing. Thrombi were always detected downstream of

12 the A/V crossing, and no eye showed thrombus only at the crossing site or upstream of

13 the A/V crossing alone. Compared with the FA or fundus examination, sequential thin

14 sectioning with OCT is a more sensitive technique to visualize the thrombus in the

15 affected retinal veins. However, Seitz ${ }^{36}$ described histological findings of a case with

16 BRVO a few hours after its onset: no blood thrombus obliterated the venous lumen at the

17 A/V crossing. It remains unclear whether intravenous thrombus formation is a cause or

18 consequence of BRVO. 
2 cross-sectional study design. In addition, we must acknowledge that due to the dense

3 retinal hemorrhage or edema, it is sometimes difficult to analyze the distal side of the A/V

4 crossing in detail. We showed the morphological and functional changes in the retinal

5 vasculature at the $A / V$ crossing in eyes with BRVO (i.e., anatomical positions of crossing

6 vessels and thrombus formation and the degree of venous tortuosity and flow condition).

7 However, most of our findings were descriptive and not quantitative. Despite these

8 shortcomings, the current OCT findings suggest that BRVO would occur from 2 different

9 mechanisms, depending on the relative anatomical positions of crossing vessels. In

10 addition, we detected a clinical relevance in the presence of thrombus in eyes with acute

11 BRVO, which were significantly correlated with the retinal perfusion status (ischemic

12 BRVO). This is a small case series; therefore, OCT studies with larger sample

13 populations would be necessary to elucidate the clinical relevance of the retinal

14 vasculature of BRVO. 


\section{REFERENCES}

3 1. Wong TY, Scott IU. Clinical practice. Retinal-vein occlusion. N Engl J Med 2010;363:2135-44.

2. Jonas J, Paques M, Mones J, Glacet-Bernard A. Retinal vein occlusions. Dev Ophthalmol 2010;47:111-35.

3. Frangieh GT, Green WR, Barraquer-Somers E, Finkelstein D. Histopathologic study of nine branch retinal vein occlusions. Arch Ophthalmol 1982;100:1132-40.

4. Weinberg D, Dodwell DG, Fern SA. Anatomy of arteriovenous crossings in branch retinal vein occlusion. Am J Ophthalmol 1990;109:298-302.

5. Jefferies P, Clemett R, Day T. An anatomical study of retinal arteriovenous crossings and their role in the pathogenesis of retinal branch vein occlusions. Aust N Z J Ophthalmol 1993;21:213-7.

6. Kumar B, Yu DY, Morgan WH, et al. The distribution of angioarchitectural changes within the vicinity of the arteriovenous crossing in branch retinal vein occlusion. Ophthalmology 1998;105:424-7.

17 7. Christoffersen NL, Larsen M. Pathophysiology and hemodynamics of branch retinal vein occlusion. Ophthalmology 1999;106:2054-62. 
1 8. Yamaike N, Tsujikawa A, Ota M, et al. Three-dimensional imaging of cystoid macular edema in retinal vein occlusion. Ophthalmology 2008;115:355-62 e2.

9. Murakami T, Tsujikawa A, Ohta M, et al. Photoreceptor status after resolved macular edema in branch retinal vein occlusion treated with tissue plasminogen activator. Am J Ophthalmol 2007;143:171-3.

10. Tsujikawa A, Sakamoto A, Ota M, et al. Serous retinal detachment associated with retinal vein occlusion. Am J Ophthalmol 2010;149:291-301 e5.

11. Yamaguchi Y, Otani T, Kishi S. Serous macular detachment in branch retinal vein occlusion. Retina 2006;26:1029-33.

12. Ogino K, Murakami T, Tsujikawa A, et al. Characteristics of optical coherence tomographic hyperreflective foci in retinal vein occlusion. Retina 2012;32:77-85.

13. Spaide RF, Lee JK, Klancnik JK Jr, Gross NE. Optical coherence tomography of branch retinal vein occlusion. Retina 2003;23:343-7.

14. Lerche RC, Schaudig U, Scholz F, et al. Structural changes of the retina in retinal vein occlusion--imaging and quantification with optical coherence tomography. Ophthalmic Surg Lasers 2001;32:272-80.

17 15. Ota M, Tsujikawa A, Murakami T, et al. Association between integrity of foveal photoreceptor layer and visual acuity in branch retinal vein occlusion. $\mathrm{Br} \mathrm{J}$ 
Ophthalmol 2007;91:1644-9.

16. Ota M, Tsujikawa A, Murakami T, et al. Foveal photoreceptor layer in eyes with persistent cystoid macular edema associated with branch retinal vein occlusion. Am J Ophthalmol 2008;145:273-80.

17. Kurashige $\mathrm{Y}$, Tsujikawa $\mathrm{A}$, Murakami $\mathrm{T}$, et al.Changes in visual acuity and foveal photoreceptor integrity in eyes with chronic cystoid macular edema associated with retinal vein occlusion. Retina in press.

18. Brezinski ME, Tearney GJ, Bouma BE, et al. Optical coherence tomography for optical biopsy. Properties and demonstration of vascular pathology. Circulation 1996;93:1206-13.

19. Wehbe H, Ruggeri M, Jiao S, et al. Automatic retinal blood flow calculation using spectral domain optical coherence tomography. Opt Express 2007;15:15193-206.

20. Kriechbaum K, Bolz M, Deak GG, et al. High-resolution imaging of the human retina in vivo after scatter photocoagulation treatment using a semiautomated laser system. Ophthalmology 2010;117:545-51.

21. Riva CE, Grunwald JE, Sinclair SH, Petrig BL. Blood velocity and volumetric flow rate in human retinal vessels. Invest Ophthalmol Vis Sci 1985;26:1124-32. 
1 22. Mason C, Markusen JF, Town MA, et al. Doppler optical coherence tomography for measuring flow in engineered tissue. Biosens Bioelectron 2004;20:414-23.

3 23. Yu L, Chen Z. Doppler variance imaging for three-dimensional retina and choroid angiography. J Biomed Opt 2010;15:016029.

5 24. Proskurin SG, He Y, Wang RK. Doppler optical coherence imaging of converging flow. Phys Med Biol 2004;49:1265-76.

25. The Eye Disease Case-control Study Group. Risk factors for branch retinal vein occlusion. Am J Ophthalmol 1993;116:286-96.

9 26. Osterloh MD, Charles S. Surgical decompression of branch retinal vein occlusions. Arch Ophthalmol 1988;106:1469-71.

11 27. Staurenghi G, Lonati C, Aschero M, Orzalesi N. Arteriovenous crossing as a risk factor in branch retinal vein occlusion. Am J Ophthalmol 1994;117:211-3.

13 28. Zhao J, Sastry SM, Sperduto RD, et al. The Eye Disease Case-Control Study Group. Arteriovenous crossing patterns in branch retinal vein occlusion. Ophthalmology 1993;100:423-8. centralis: hypotheses concerning its role in the pathogenesis of macular hole and foveomacualr retinoschisis. Arch Ophthalmol 1999;117:821-3. 
$130 . \quad$ Marmor MF. Mechanisms of fluid accumulation in retinal edema. Doc Ophthalmol

3 31. Hamid S, Mirza SA, Shokh I. Anatomic pattern of arteriovenous crossings in

branch retinal vein occlusion. J Pak Med Assoc 2008;58:233-6.

32. Rehak J, Rehak M. Branch retinal vein occlusion: pathogenesis, visual prognosis, and treatment modalities. Curr Eye Res 2008;33:111-31.

33. Opremcak EM, Bruce RA. Surgical decompression of branch retinal vein occlusion via arteriovenous crossing sheathotomy: a prospective review of 15 cases. Retina 1999;19:1-5.

34. Mason J 3rd, Feist R, White M Jr, et al. Sheathotomy to decompress branch retinal vein occlusion: a matched control study. Ophthalmology 2004;111:540-5.

35. Horio N, Horiguchi M. Effect of arteriovenous sheathotomy on retinal blood flow and macular edema in patients with branch retinal vein occlusion. Am J Ophthalmol 2005;139:739-40.

36. Seitz R. (Blodi F.C., translator) The "crossing phenomenon." In The Retinal Vessels. St. Louis, C.V. Mosby 1964:20-74. 
Table 1. Characteristics of patients with acute branch retinal vein occlusion

Age (years)

Gender (women/men)

Duration of symptoms until examination (weeks)

Visual acuity (logMAR)

Foveal thickness $(\mu \mathrm{m})$

Foveal cystoid spaces

Hemorrhage within foveal cystoid spaces

Foveal serous retinal detachment

Foveal subretinal hemorrhage

Retinal perfusion status (nonischemic/ischemic)
$66.3 \pm 11.9$

$13 / 12$

$$
\begin{gathered}
10.0 \pm 7.1 \\
0.33 \pm 0.32
\end{gathered}
$$

$577.6 \pm 220.5$

$23(92 \%)$

$18(72 \%)$

$16(64 \%)$

$12(48 \%)$

$13 / 12$

$\log M A R=$ logarithm of minimal angle of resolution. 
Table 2. Comparisons of optical coherence tomography findings between 2 groups classified by the presence or absence of thrombus in the affected veins

\begin{tabular}{lccc}
\hline & Thrombus $(+)$ & Thrombus $(-)$ & $P$ value \\
\hline Visual Acuity (logMAR) & $0.38 \pm 0.34$ & $0.15 \pm 0.12$ & 0.210 \\
Duration of symptoms (weeks) & $11.1 \pm 7.2$ & $4.1 \pm 1.6$ & 0.070 \\
Foveal thickness $(\mu \mathrm{m})$ & $592.4 \pm 233.7$ & $500.3 \pm 124.8$ & 0.456 \\
Height of serous retinal detachment $(\mu \mathrm{m})$ & $155.2 \pm 126.8$ & $37.3 \pm 43.1$ & 0.068 \\
Foveal cystoid spaces (+/-) & $18 / 3$ & $4 / 0$ & 0.420 \\
Hemorrhage within foveal cystoid spaces (+/-) & $15 / 6$ & $3 / 1$ & 0.884 \\
Foveal serous retinal detachment $(+/-)$ & $14 / 7$ & $2 / 2$ & 0.524 \\
Foveal subretinal hemorrhage $(+/-)$ & $11 / 10$ & $4 / 0$ & 0.315 \\
Retinal perfusion status (nonischemic/ischemic) & $9 / 12$ & $4 / 0$ & 0.036 \\
\hline
\end{tabular}




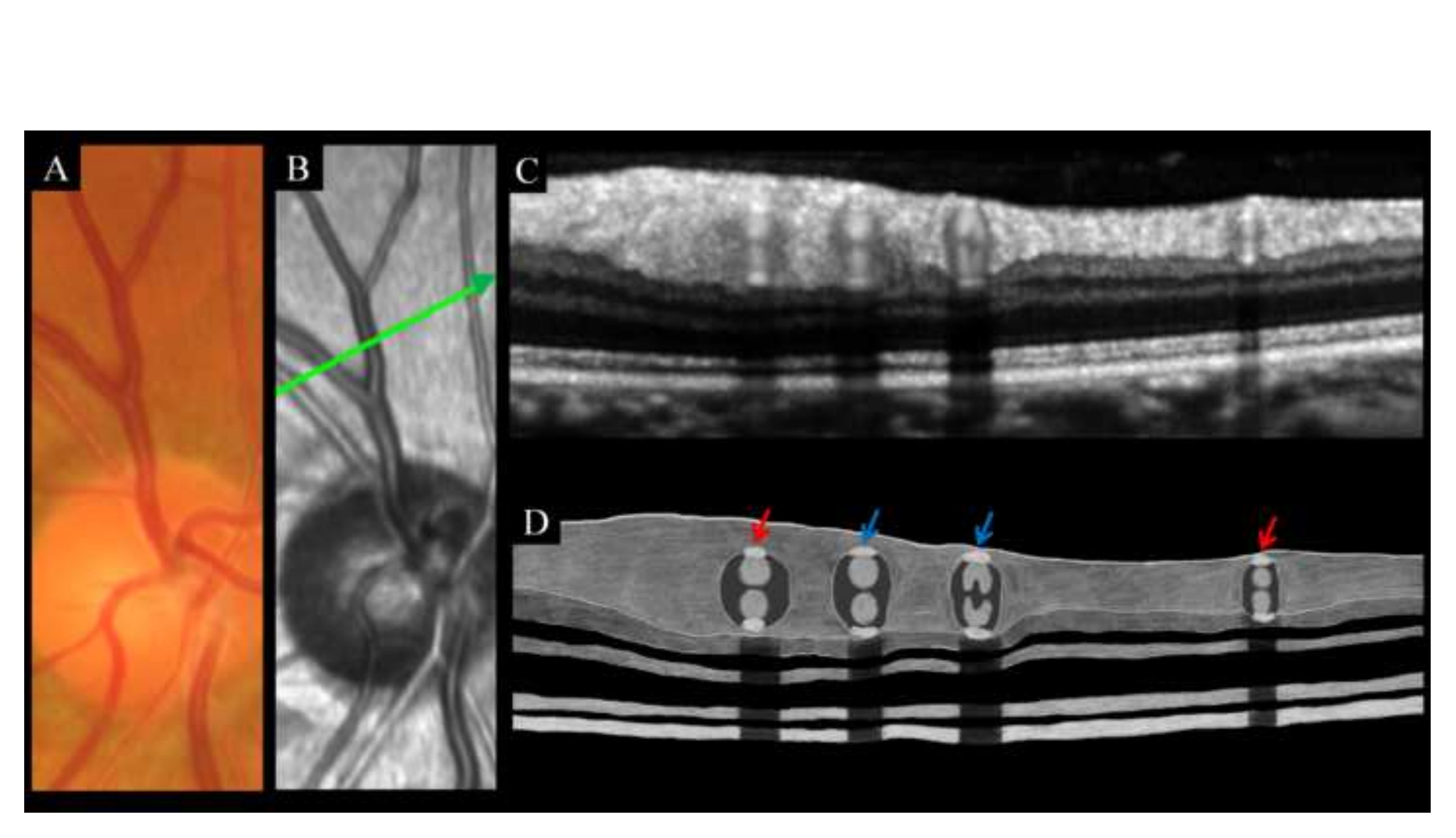

Click here to download high resolution image

\footnotetext{
.
}

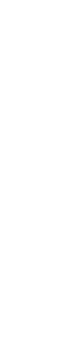
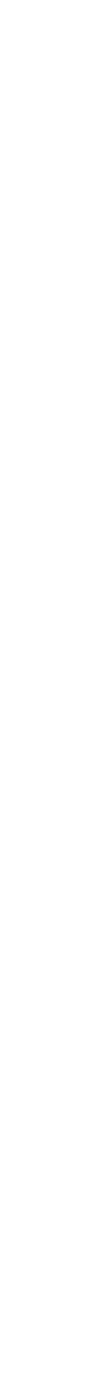

(1)
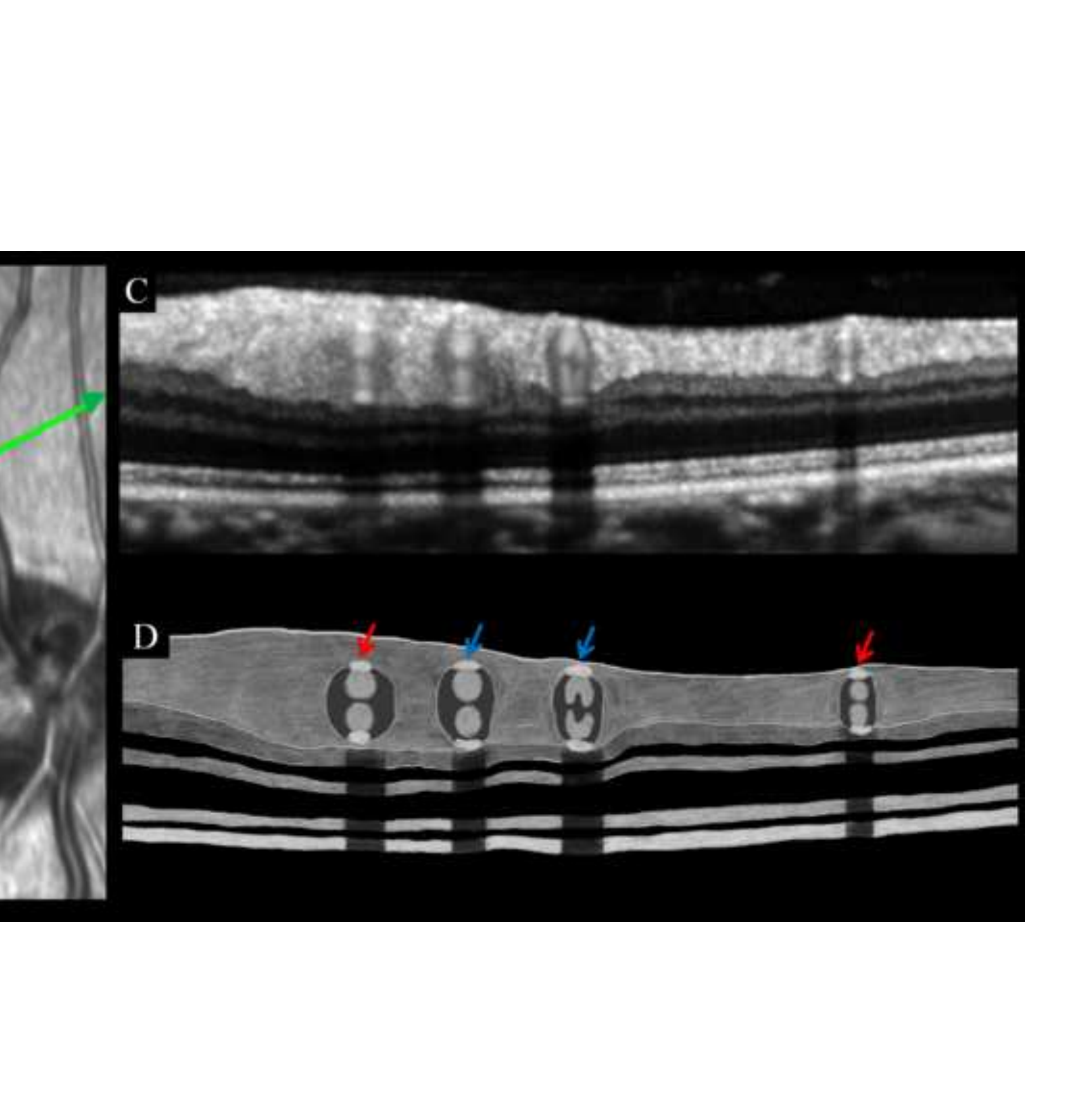


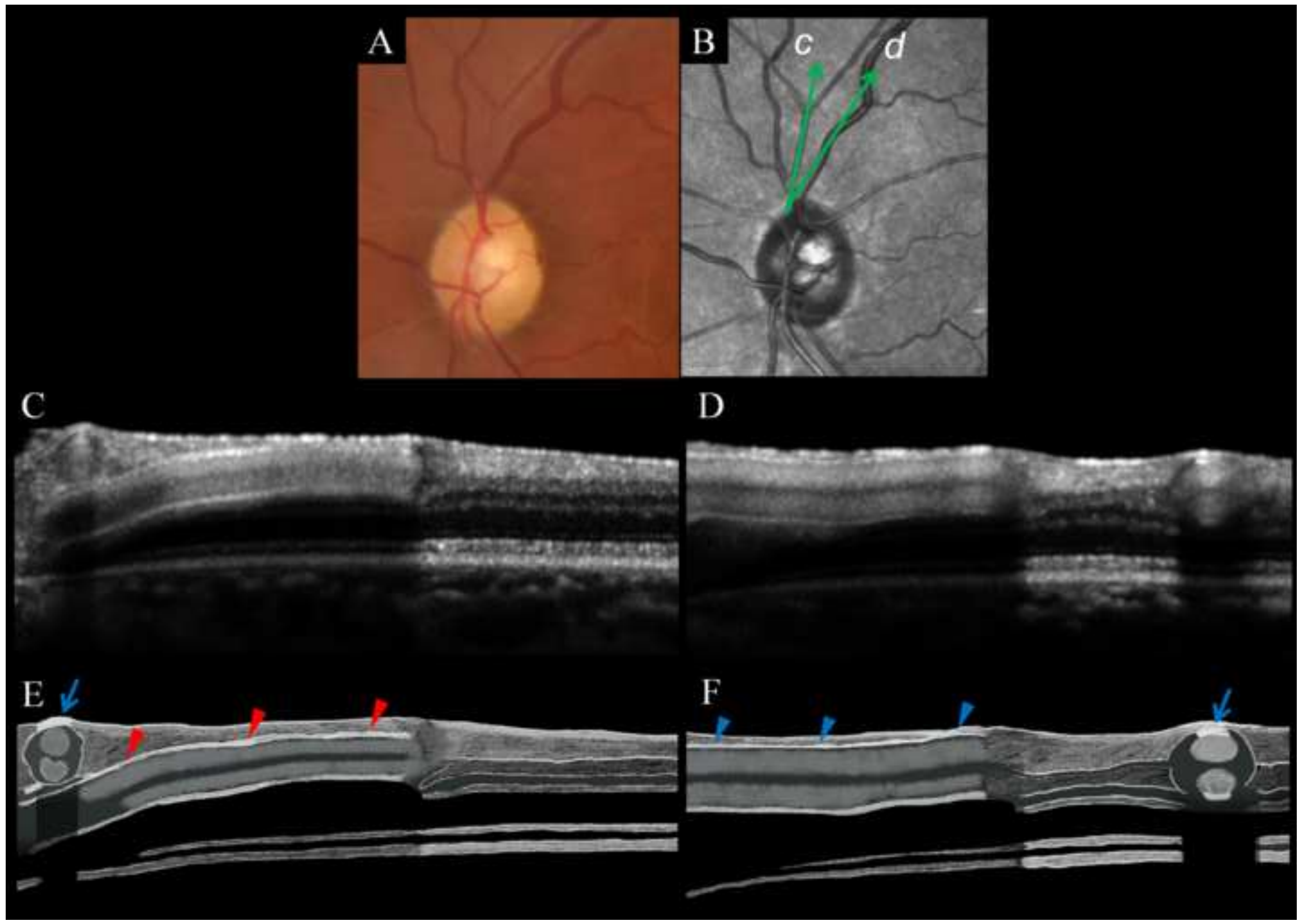

$\mathrm{C}$

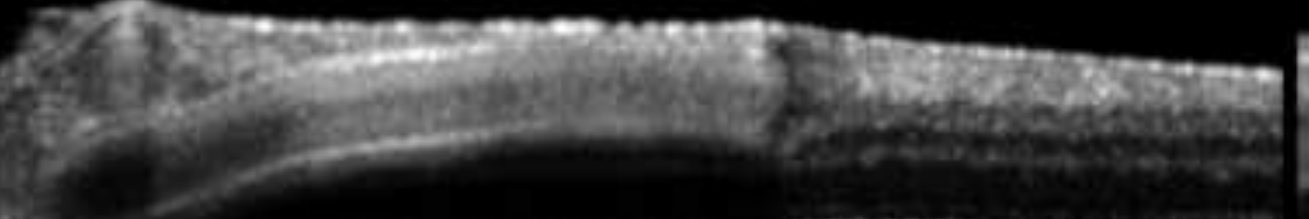

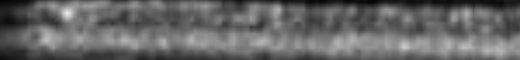

$\mathrm{E}$ 


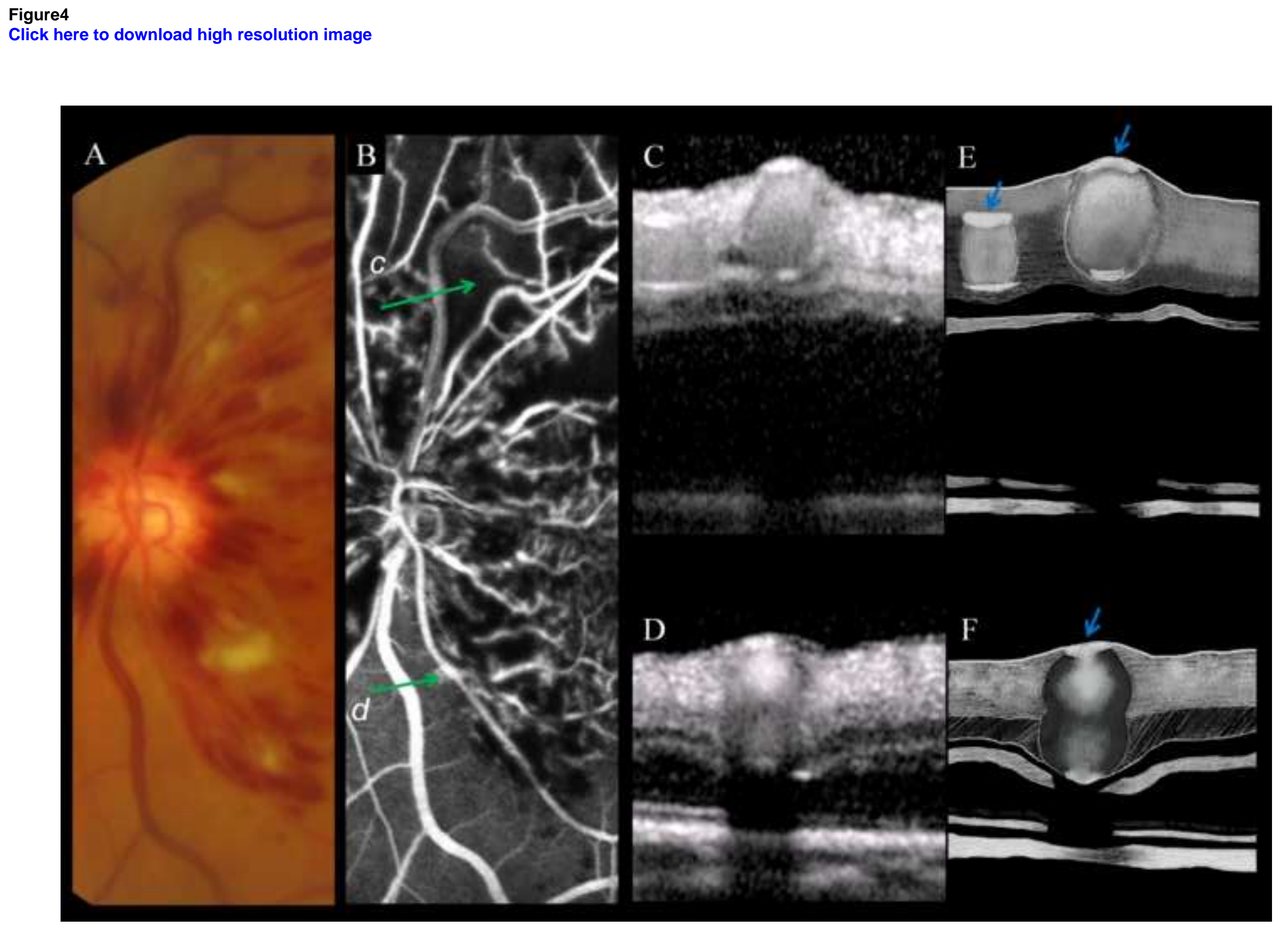

$$
\text { . }
$$
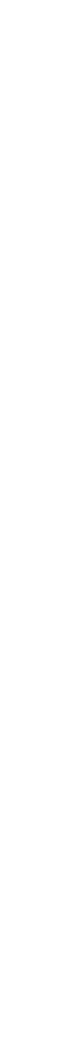


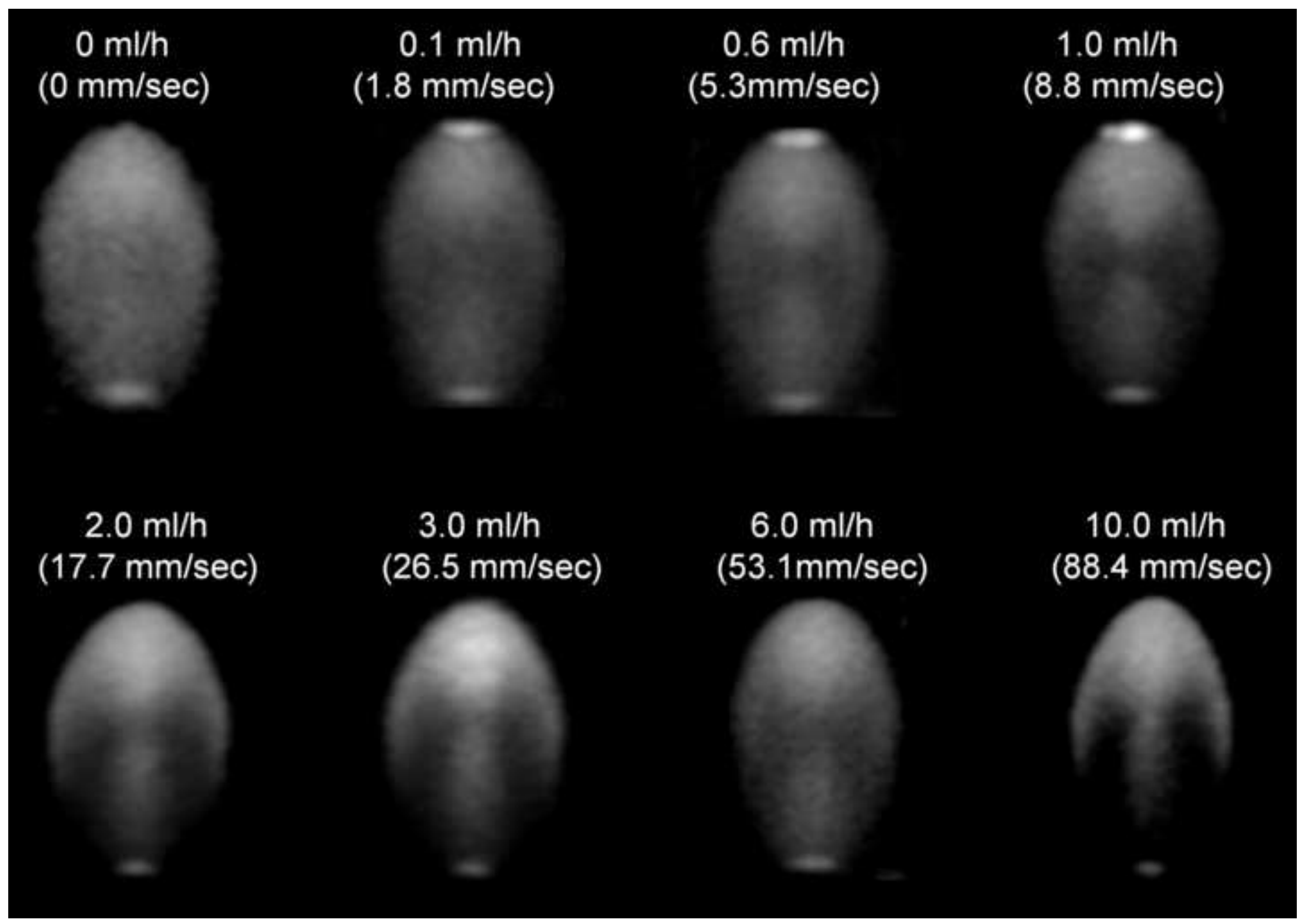




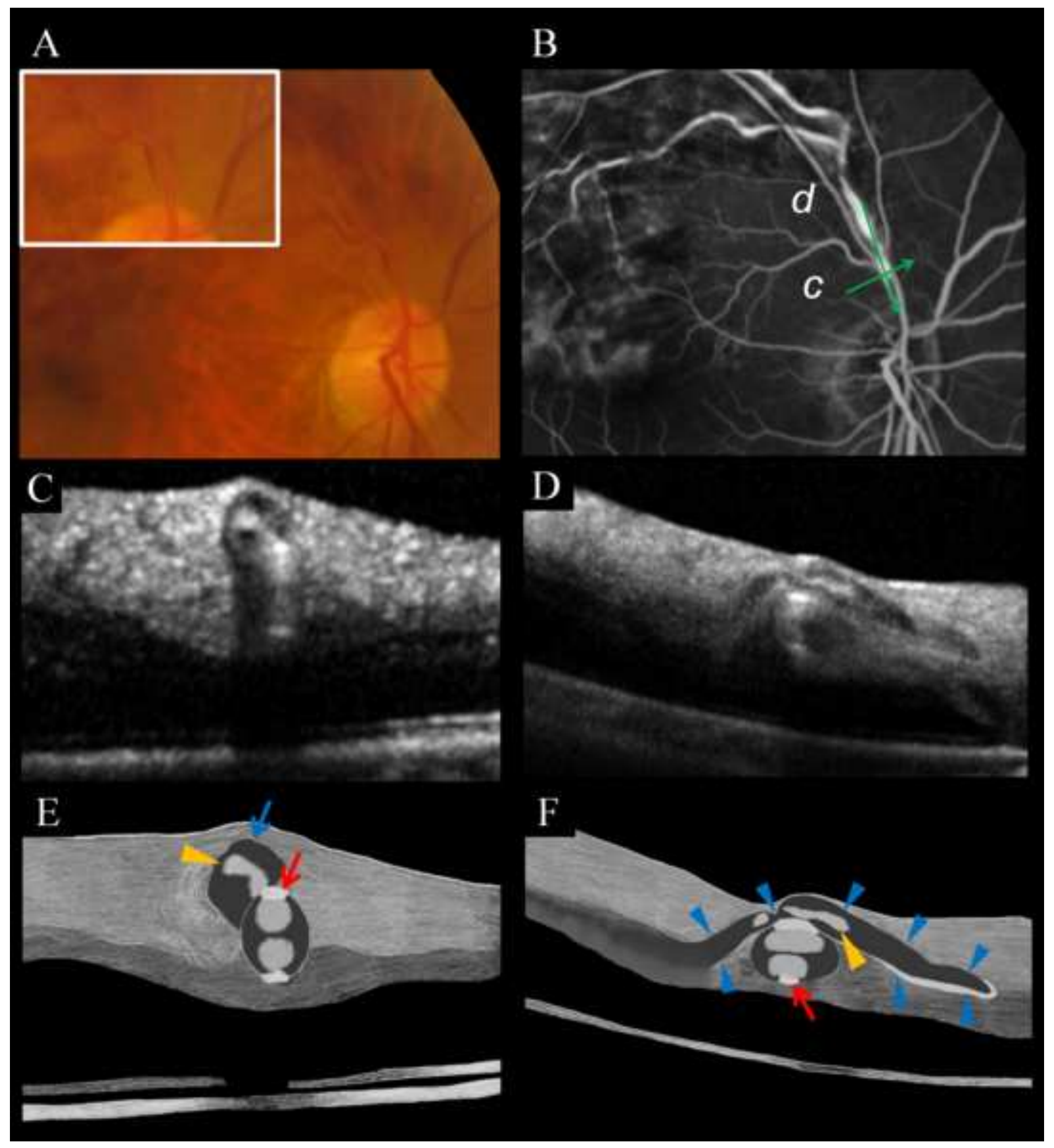




\section{Figure legends}

Figure 1. Cross-section of optical coherence tomography (OCT) in normal retinal vessels. Fundus photograph $(\mathbf{A})$ and infrared image $(\mathbf{B})$ of an unaffected contralateral eye. (C) An OCT image shows cross-sections of the retinal vessels along the green arrow. Major retinal vessels are seen as oval-shaped configurations with 4 distinctive hyperreflectivities. The top and bottom of the vessel walls show the innermost and outermost hyperreflectivities. The reflectivity from the arterial walls is higher, compared with that from venous walls. All retinal vessels with a physiological blood flow showed

10 inside hourglass-shaped hyperreflectivities. The internal paired hyperreflectivities show a double "c" pattern in the convergent veins. (D) An illustration of Figure 1C. Red arrows indicate arteries and blue arrows indicate veins.

Figure 2. Longitudinal section of optical coherence tomography (OCT) in normal retinal

15 vessels. Fundus photograph $(\mathbf{A})$ and infrared image (B) of an unaffected contralateral eye. OCT images along green arrows $\boldsymbol{c}$ and $\boldsymbol{d}$ show longitudinal sections of the major retinal artery $(\mathbf{C})$ and vein $(\mathbf{D})$. Consistent with the cross-sections of the major retinal vessels, retinal vessels show 4 hyperreflective bands. The innermost and outermost 
bands are derived from the vessel walls, and the 2 intermediate bands are derived from the bloodstream. (E, F) Illustrations of Figure 2C and 2D. Red arrows indicate arteries and blue arrows indicate veins.

$5 \quad$ Figure 3. Optical coherence tomography (OCT) images of the retinal vein without blood flow. (A) Fundus photograph of a sheathed vein associated with old branch retinal vein occlusion. (B) Fluorescein angiogram shows no blood flow in the sheathed retinal vein. OCT images along green arrows $\boldsymbol{c}$ and $\boldsymbol{d}$ show a cross-section $(\mathbf{C})$ and a longitudinal section (D) of the occluded retinal vein. Vessel lumen is totally hyperreflective. (E, F)

10 Illustrations of Figure 3C and 3D. Red arrows indicate arteries and blue arrows indicate veins.

Figure 4. Cross-sections of retinal veins with physiological and disturbed blood flow. Fundus photograph $(\mathbf{A})$ and fluorescein angiogram $(\mathbf{B})$ of an eye with hemi-central retinal

15 vein occlusion. Optical coherence tomography images along green arrows $\boldsymbol{c}$ and $\boldsymbol{d}$ show the cross-sections of the affected upper veins (C) and an unaffected lower vein (D). (C) Affected upper veins with disturbed blood flow show homogeneous internal reflectivity. (D) Unaffected lower vein with physiological blood shows innermost and outermost 
hyperreflectivities from the venous wall and internal paired hyperreflectivities from blood

flow. (E, F) Illustrations of Figure 4C and 4D. Blue arrows indicate veins.

Figure 5. Cross-sectional images of the blood flowing in the fine glass tube at different

5 flow velocities obtained with Spectralis HRA+OCT. Cross-section of the blood within the glass tube without flow (at $0 \mathrm{~mm} / \mathrm{sec}$ ) shows homogeneous reflectivities. When the blood flow velocity was increased, the internal reflectivity became heterogeneous and hourglass-shaped hyperreflectivities were detected within the glass tube. At unphysiological blood flow velocity $(88.4 \mathrm{~mm} / \mathrm{s})$, the internal reflectivity from the posterior

10 part of the lumen disappeared.

Figure 6. Branch retinal vein occlusion at the arterial overcrossing. Fundus photograph (A) and fluorescein angiogram (B) of the branch retinal vein occlusion. Fluorescein angiogram confirms the arterial overcrossing at the affected crossing site.

15 (C) Sectional image was obtained along the green arrow by using optical coherence tomography. The vein appears to run deep in the retinal layers below the overcrossing artery and appears to approach the junction between the inner and outer segments of the photoreceptor (IS/OS). The lumen of the vein at the crossing site is maintained. 
An illustration of Figure 6C. Red arrows indicate arteries and blue arrows indicate veins.

Figure 7. Branch retinal vein occlusion occurred at the venous overcrossing. Fundus photograph $(\mathbf{A})$ and fluorescein angiogram $(\mathbf{B})$ of branch retinal vein occlusion. $\quad$ (C, D)

5 Sectional image along green arrows $\boldsymbol{c}$ and $\boldsymbol{d}$ were obtained with optical coherence tomography. (C) Cross sectional image confirmed the venous overcrossing at the affected crossing site. (D) The retinal vein appears to be compressed and choked between the internal limiting membrane and the arterial wall at the crossing. A thrombus was seen within the lumen at the crossing site. (E, F) Illustrations of Figure

10 7C and 7D. Red arrows indicate arteries and blue arrows indicate veins. Yellow arrows indicate the thrombus.

Figure 8. Thrombi within the vein affected with branch retinal vein occlusion. Fundus photograph $(\mathbf{A})$ and fluorescein angiogram $(\mathbf{B})$ of branch retinal vein occlusion. $\quad$ (C-E)

15 Sectional images along green arrows $\boldsymbol{c}, \boldsymbol{d}$, and $\boldsymbol{e}$ were obtained with optical coherence tomography. (C, D) Retinal artery at the crossing site shows a physiological appearance with internal paired hyperreflectivities. Retinal vein proximal to the affected crossing shows thrombus formation without physiological appearance. (E) Longitudinal section 
Changes in Retinal Vessels due to BRVO. Muraoka Y, et al. Page 5

of the retinal vein shows internal thrombus formation downstream of the affected crossing.

(E-G) Illustrations of Figure 8C, 8D and 8E. Red arrows indicate arteries and blue arrows indicate veins. Yellow arrows indicate thrombus. 